\title{
Search for Extreme Energy Cosmic Rays with the TUS orbital telescope and comparison with ESAF
}

\author{
Mario Bertaina ${ }^{1 *}$, Antonella Castellina ${ }^{2}$, Roberto Cremonini ${ }^{1}$, Francesco Fenu ${ }^{1}$, Pavel Klimov $^{3}$, Anthony Salsi ${ }^{1,4}$, Sergey \\ Sharakin $^{3}$, Kenji Shinozaki ${ }^{1}$, and Mikhail Zotov ${ }^{3}$ On behalf of the TUS and JEM-EUSO Collaborations \\ ${ }^{1}$ Department of Physics, University of Turin - Italy \\ ${ }^{2}$ National Institute for Astrophysics (INAF) - OATo, Turin - Italy \\ ${ }^{3}$ Lomonosov Moscow State University, Skobeltsyn Institute of Nuclear Physics, Moscow - Russia \\ ${ }^{4}$ Université Côte d'Azur, Observatoire de la Côte d'Azur, Laboratoire Lagrange, Nice - France
}

\begin{abstract}
The Tracking Ultraviolet Setup (TUS) detector was launched on April 28, 2016 as a part of the scientific payload of the Lomonosov satellite. TUS is a pathfinder mission for future space-based observation of Extreme-Energy Cosmic Rays (EECRs, E $>5 \times 10^{19} \mathrm{eV}$ ) with experiments such as K-EUSO. TUS data offer the opportunity to develop strategies in the analysis and reconstruction of the events which will be essential for future space-based missions. During its operation, TUS has detected about 80 thousand events which have been subject to an offline analysis to select among them those that satisfy basic temporal and spatial criteria of EECRs. A few events passed this first screening. In order to perform a deeper analysis of such candidates, a dedicated version of ESAF (EUSO Simulation and Analysis Framework) code as well as a detailed modelling of TUS optics and detector are being developed.
\end{abstract}

\section{Introduction}

Ultra-High-Energy Cosmic Rays (UHECRs) allow studying phenomena that occur at energies currently inaccessible by man-made accelerators. Although work in recent years has shed light on several characteristics of these particles with $E>10^{18} \mathrm{eV}$, their origin, nature and acceleration mechanisms are still unclear. A more detailed understanding of UHECRs can help clarifying aspects related to the highest energy astrophysics and particle physics mechanisms, potentially addressing yet-unknown phenomena, such as Big Bang cosmology or Lorentz invariance violation. The difficulty in unraveling these problems lies in the extremely low flux of particles above $5 \times 10^{19} \mathrm{eV}$, the so called EECRs, which requires the construction of very large ground based detectors, such as the Pierre Auger Observatory (PAO) in the Southern Hemisphere [1] and Telescope Array (TA) [2] in the Northern one. Space-based observations have a potential for an increase in statistics, up to orders of magnitude and would be able to cover the whole sky uniformly, allowing for a direct comparison of spectra and arrival directions of EECRs on the celestial sphere.

J. Linsley and R. Benson [3] were the first to propose the fluorescent registration of Extensive Air Showers (EAS) using a UV telescope on board a satellite. This idea has been developed in a number of projects. In the USA, there were the proposals of OWL-AIRWATCH [46], and the first EUSO (ESA) design was suggested in Europe $[7,8]$. In parallel, in Russia, detectors that use con-

*e-mail: bertaina@to.infn.it centrator mirrors for collecting fluorescence light (TUS and KLYPVE) were proposed and developed $[9,10]$ with TUS, the first orbital detector of UHECRs, being launched aboard the Lomonosov satellite on April 28, 2016. All these initiatives led to the development of the JEMEUSO mission [11] which subsequently evolved into the JEM-EUSO program: Joint Experiments Mission for Extreme Universe Space Observatory. The JEM-EUSO program includes experiments on ground (EUSO-TA [12]), on stratospheric balloons (EUSO-Balloon [13], EUSOSPB $[14,15])$ and in space (Mini-EUSO [17], TUS [16]) with the final goal to have large-size missions for the detection of EECRs from space, such as K-EUSO [18] and POEMMA [19].

The principle of observation of all the conceived so far space-based missions relies on the detection of UV light emitted by isotropic fluorescence of atmospheric nitrogen excited by the EASs in the Earth's atmosphere and forward-beamed Cherenkov radiation reflected from the Earth's surface or dense cloud tops. The design of a spacebased telescope for UHECR observation has strong constraints on power, mass, size and data transmission bandwidth and requires the development of a number of novel technologies, from optics to sensors, front-end and readout electronics. The various experiments and pathfinder missions, such as TUS, are essential in pursuing this effort. 

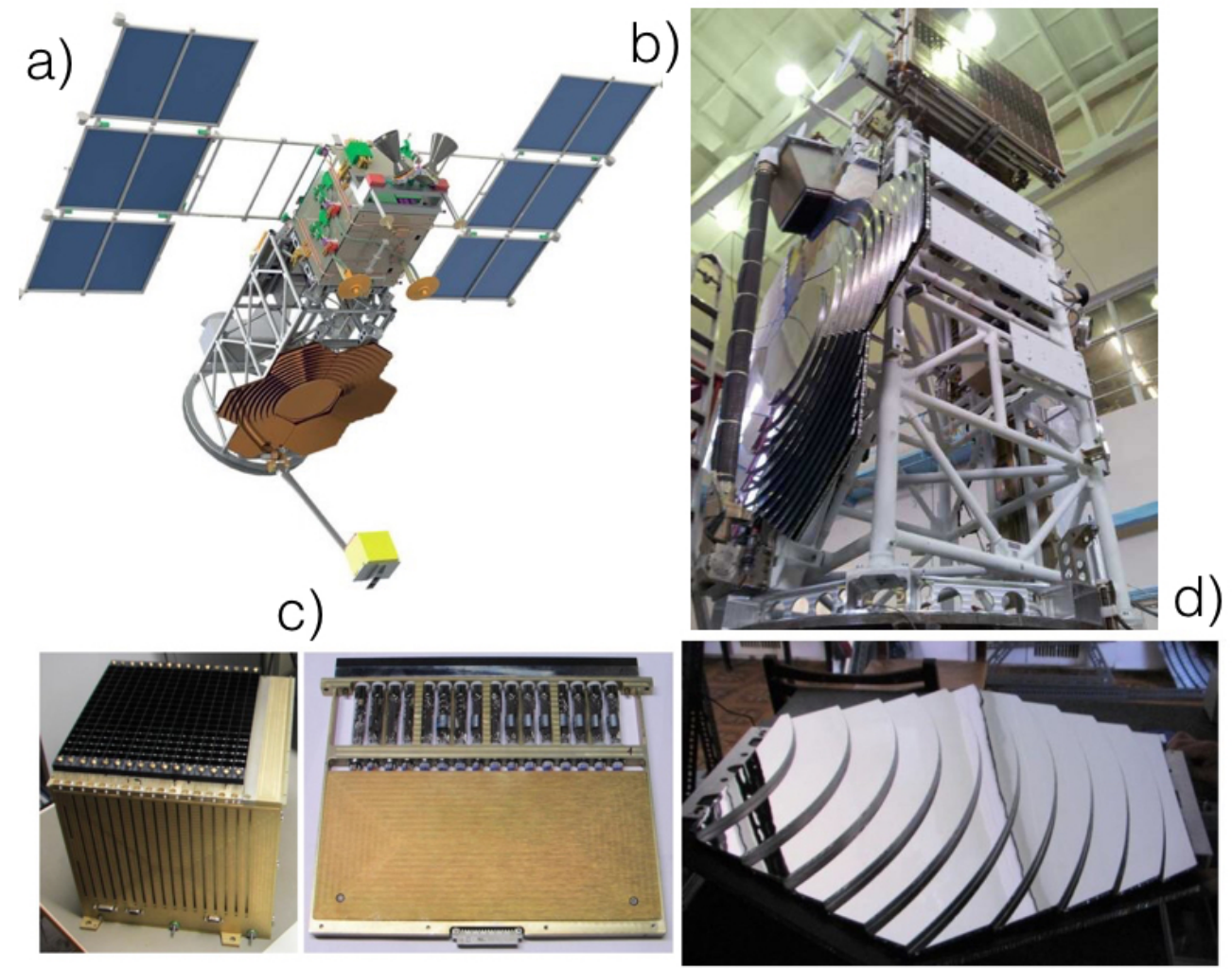

Figure 1. a) Artist's view of the TUS detector on board the Lomonosov satellite; b) TUS on board the Lomonosov satellite during preflight preparations at cosmodrome Vostochny; c) TUS Focal Surface; d) TUS Fresnel mirror

\section{The TUS detector on board the Lomonosov satellite}

The TUS detector is the first attempt to measure UHECR fluorescent light from space. It was launched on April 28 , 2016, on a polar sun-synchronous orbit with inclination of $97.3^{\circ}$, period of $\sim 94 \mathrm{~min}$, and altitude about $500 \mathrm{~km}$. TUS was operated regularly till December 2017, when the Lomonosov satellite faced some technical problems that did not allow transmitting experimental data to Earth. The TUS detector consists of two main parts: a parabolic mirror-concentrator of the Fresnel type and a square-shaped 256-pixel photodetector in the focal plane of the mirror (see Fig. 1).

The mirror has an area of about $2 \mathrm{~m}^{2}$ with a focal distance of $1.5 \mathrm{~m}$. A pixel field of view equals $10 \mathrm{mrad}$, which results in spatial resolution of $5 \mathrm{~km}$, and the overall TUS Field of View (FoV) of approximately $80 \mathrm{~km} \times 80 \mathrm{~km}$ at the sea level. Each pixel of the TUS photodetector is a Hamamatsu R1463 photomultiplier tube. Light guides with square entrance apertures $(15 \mathrm{~mm} \times 15 \mathrm{~mm})$ and circular outputs were employed to fill uniformly the detector's FoV. Each pixel has a black blind $1 \mathrm{~cm}$ above the light guide to protect it from stray light. An UV filter of $13 \mathrm{~mm}$ diameter and $2.5 \mathrm{~mm}$ thickness is placed in front of each PMT. The pixels are grouped in 16 identical photodetector modules. Each cluster has its own digital data processing system for the first-level trigger, based on a Xilinx Field-Programmable Gate Array (FPGA), and a high voltage power supply, controlled by the FPGA. The central processor board gathers information from all modules, controls their operation, and implements the second-level trigger algorithm, see [16, 20] for details.

The TUS electronics can operate in four modes intended for detecting various fast optical phenomena in the atmosphere at different time scales with different time sampling. The main mode is aimed at registering EECRs and has a time sampling of $0.8 \mu \mathrm{s}$. This mode is also efficient for short Transient Luminous Events (TLEs) measurements, for example, elves. Slower modes have time sampling of $25.6 \mu \mathrm{s}, 0.4 \mathrm{~ms}$ (for studying TLEs of different kinds slower than elves: sprites, blue jets, gigantic jets, etc.). An even slower mode of $6.6 \mathrm{~ms}$ is devoted to the detection of micro-meteors, and thunderstorm activity at a longer time scale. The four operational modes cannot be run in parallel, therefore, the selected mode has to be fixed at the start of a run. Waveforms in each mode consist of 256 time samples. The first level trigger decision is based on a comparison of the simple moving average 
of analog-to-digital converter (ADC) counts calculated for each pixel with a threshold level that depends on the mean value of the background noise. At the second level trigger, the geometry and number of hit pixels are analyzed. In case of EASs and meteors it searches for a track, i.e., adjacent pixels lined up within a certain time. The most updated status of the analysis of TUS data and results using all four operational modes can be found in [21, 22].

\section{Data Analysis of EECR mode}

During its operation, TUS detected about $8 \times 10^{4}$ events that have been subject to an offline analysis to select among them those satisfying basic temporal and spatial criteria of EECRs. A few events passed this first screening. In order to perform a deeper analysis of such candidates, a dedicated version of ESAF (EUSO Simulation and Analysis Framework) [23] as well as a detailed modeling of TUS optics and detector are being developed.

The search for EECR signals in the data requires different steps. First of all, the signal selection in the ADC time track is performed. In one of the approaches, the signal significance is used. The signal in one packet of 256 time ticks is divided in five parts, and the average SNR value is determined in each time window. If there is a window with an average SNR $>2$, the pixel is assumed to have a signal excess. This procedure is applied for each pixel. In case of an EECR event, it is expected that several pixels show similar behaviour with a signal excess shifted in time in a consistent way along the track (see Fig. 2). It is important anyway to remember that the tracks are typically short due to the large pixel size at ground $(\sim 5 \mathrm{~km})$. Simulation of EASs should reproduce similar time evolution of the signal, as well as the light intensity in the pixels.

Other important aspects to be considered are the atmospheric conditions as well as the geographical area of the event. For this reason, the data from satellites and MERRA-2 model [24] have been used to understand the possible presence of high clouds which would hamper the detection of EECRs as well as lightning activity, which could be responsible for short and bright light pulses. The DMSP [25] (Defense Meteorological Satellite Program) data have been used in parallel to check the possible presence of anthropogenic lights, which raise the energy threshold of detectable EECRs and can indicate the possible presence or artificial pulsed lights, such as laser shots, strobe lights, etc. near airports and other places. Several triggers are indeed coming from areas near airports [26]. A more detailed description of the most interesting EECR candidates detected by TUS can be found in [27].

Light sources of different origin such as laser pulses have been simulated as a part of an analysis of fake EECR signals. Such pulses could be directed towards TUS if generated by a laser beam on ground, but could also point downward like in case of an airplane lidar. First simulations of waveforms obtained with laser pulses look different from those of EAS candidates, but more simulations and analyses are needed.

An upper limit on the exposure around the threshold energy range has been derived by looking at the time difference between consecutive events collected in EAS mode and by considering only moonless nights. The number of triggered events reduces to $\sim 23 \%$ when this selection is applied. It is important to remind that in the TUS acquisition system the sensitivity of the PMTs is decreased by the HV control system, therefore, the instrument can be operated in any light condition, even though the sensitivity starts at higher energies during moon light conditions. We notice that the peak of the time difference between consecutive events is located around $53 \mathrm{~s}$. This has to be assumed as the dead time of the instrument. Other penalty factors come from the presence of high clouds that block the detection of the events, the presence of artificial lights, mainly on continents as well as lightnings, aurorae, etc. Taking into account these factors by assuming similar weight as in JEM-EUSO [28], leads to a value of $\sim 1360$ $\mathrm{km}^{2} \mathrm{sr}$ yr. This value does not take into account detector inefficiencies, therefore, this estimation can be considered as an upper limit on the real value, but defines as a first level of approximation the correct order of magnitude of the exposure. This is important in view of the number of EECR events that potentially could have been detected by TUS as a function of its energy threshold according to the EECR flux based on the Auger and TA measurements.

\section{Simulation Developments}

ESAF is the simulation software developed in the framework of the ESA-EUSO mission. This software was developed as the mission simulation software to take care of the simulation of all the relevant processes from the shower simulation until the event reconstruction. ESAF is subdivided in two main parts: Simulation and Reconstruction. The simulation framework is meant to simulate all the physical processes which are related to the shower development, the light production and propagation, the detector and eventually the telemetry. Several shower simulators are implemented in ESAF, following parametric and Monte Carlo approaches. As parametric generator, the Greisen-Il'ina-Linsley (GIL) function [30] is used to reproduce the profile for hadronic EASs:

$$
N_{e}=\frac{E}{1.45 \cdot 10^{9} e V} \cdot e^{t-t_{\max }-2 \cdot t \cdot \ln \frac{2}{1+\frac{I_{\max }}{t}}}
$$

whereas $N_{e}$ is the shower size, $t=\frac{X}{37.59 / \mathrm{cm}^{2}}$ is an expression of the slant depth in units of mean interaction length of protons in air $\left(37.5 \mathrm{~g} / \mathrm{cm}^{2}\right), E$ is the primary energy and A the atomic mass of the particle. Furthemore, $t_{\max }$ expresses the slant depth of the maximum of the shower (after the subtraction of the first interaction length) in units of mean interaction length and it has a form like:

$$
t_{\max }=1.7+0.76 \cdot\left(\ln \frac{E}{8.1 \cdot 10^{7} e V}-\ln A\right)
$$

whereas $\mathrm{A}$ is the atomic mass of the particle. In the previous formulas the number of particles at each slant depth is univocally determined by just two parameters: the primary energy and the atomic mass of the particle. Once the first interaction point is fixed, which is 
a)

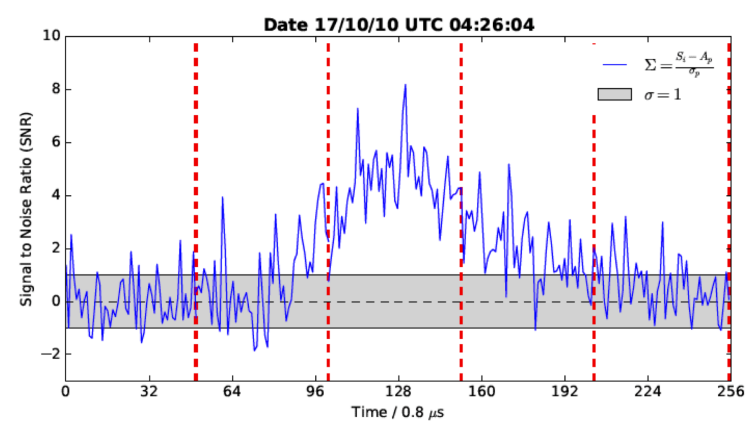

b)

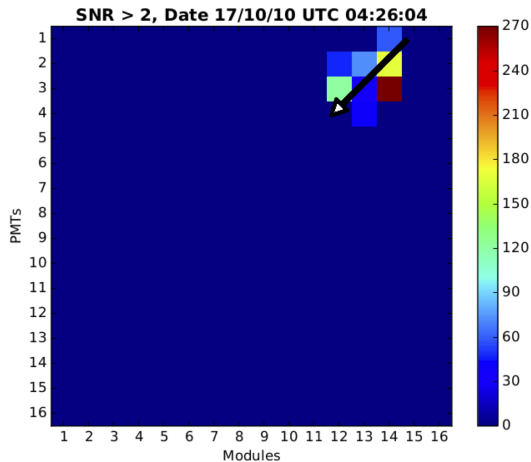

c)

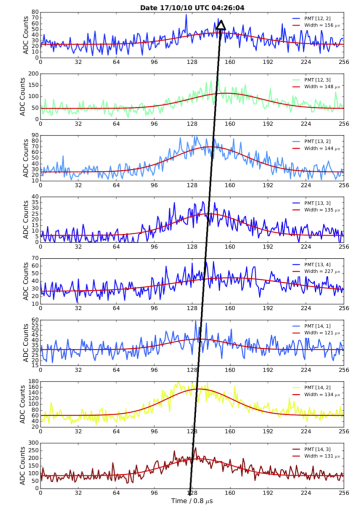

Figure 2. Data analysis of TUS event TUS171010: a) Signal selection on a pixel by requiring SNR $>2$; b) track of the pixels satisfying condition a); c) time profile of the signal in the pixels indicated in b). A gaussian fit is performed on the data to have a better description of the time evolution of the signal maximum in each pixel.
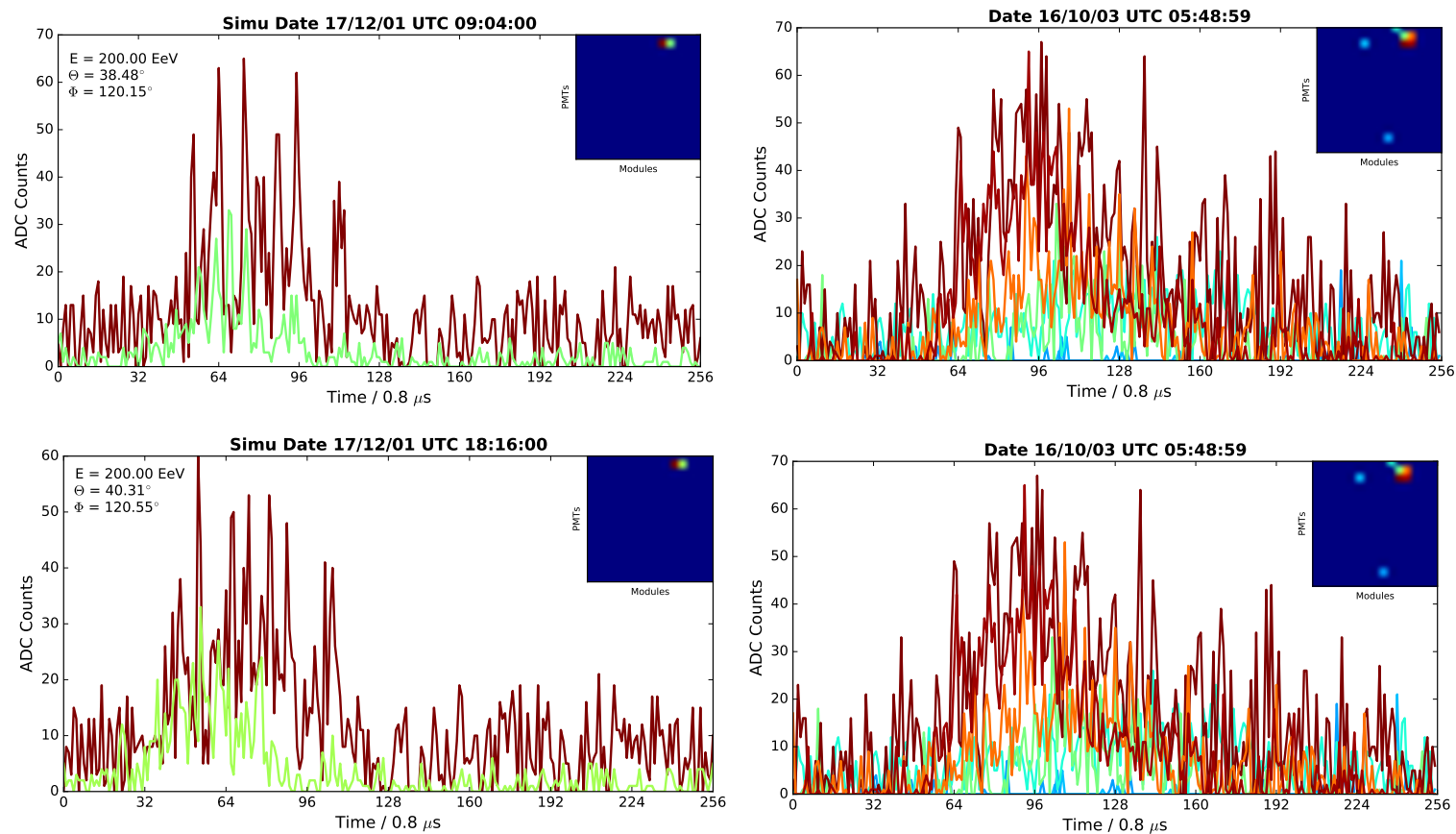

Figure 3. Simulations (left) of an experimental event (right) which has characteristics similar to an EECR event. An arbitrary response of the TUS detector is assumed to match signal intensity in simulations with those expected for a $200 \mathrm{EeV}$ event.

randomly chosen according to an exponential distribution scaled by the mean hadronic interaction length, this parameterization does not imply any oscillation from shower to shower. Both fluorescence and Cherenkov light (reflected and back-scattered) productions are taken into account in ESAF. The fluorescence spectrum is simulated according to [31]. The Cherenkov reflection is treated according to a Lambertian reflector. Therefore, all the photons are reflected diffusely due to the very irregular terrestrial surface. An albedo of 5\% is considered for water and forests. However, in case of land the value typically varies from $2 \%$ on savannah to $85 \%$ on fresh snow. All the photons are affected by Rayleigh scattering and ozone absorption. Optionally, clouds can be simulated as a constant layer of variable altitude thickness and optical depth. Nonuniform cloud coverage is also included in ESAF. Once the photons reach the detector, they are taken over by the optics module and later on the Focal Surface (FS) response is taken into account. The ESAF software was readapted for the JEM-EUSO instrument [29] and for all the other missions of the JEM-EUSO program. Currently, it is being updated to include also the response of the TUS detector.

Several optics simulation approaches have been considered in ESAF. The simplest and fastest one is a parametric simulation module that calculates analytically the position of the photon on the FS and adds a Gaussian spread around this position. This is intended to be a fast working tool to test the features of the different optics designs 
in an approximated way. This is the one which is currently used for TUS as well. Second, a full ray-trace code used in the actual optics design. This is under development also for the TUS detector and will be employed in future. Once the photons reach the FS, they are transported through the filter and the optical adapter before reaching the photocathode. All the relevant effects including geometrical losses, inefficiencies of the adapter and of filters are taken into account. A parametrization of a photomultiplier response is included in the electronics part. All the effects like quantum efficiency, dependence on the incident angle of photon, collection efficiency and cross talk are also taken into account. The signal is then amplified by a parametrized gain and the resulting output current is collected and treated by the Front End Electronics module.

The major differences of the TUS implementation compared to the original JEM-EUSO development are the optics response, which is based on a reflective system instead of a refractive one, and the electronics response based on the charge integration instead of the photoncounting approach of JEM-EUSO.

The modelling of the conversion between photoelectrons and ADC counts is in progress. This requires an absolute calibration of the detector which is being performed using data acquired in flight. Fig. 3 shows the comparison between an experimental event (right side) with two different simulations of the same event (left side) where the zenith angle is slightly modified to see the variations of the light intensity and profile. In this simulation, an arbitrary response of the TUS detector is assumed to match the signal intensity of a $200 \mathrm{EeV}$ simulated event and the experimental data.

The second aspect under development is the modelling of the optics response, more specifically the PSF of the optics. Currently a Gaussian shape is assumed, while a proper modelling of the Fresnel mirror response is under development (see Fig. 4).

\section{Conclusions}

TUS is the first orbital detector designed to be sensitive to UHECRs at the highest energies. Among the several thousand events detected by TUS, a few of them have characteristics which make them interesting to be analyzed more carefully and compared to simulations of EECRs. A new version of ESAF that includes the TUS detector is currently being developed. The comparison with simulations will be very important to understand the origin of such events. At the same time, such events offer the possibility to develop methodologies of analysis which will be employed in the next generation of missions such as KEUSO and POEMMA, and prove that space-based observatories have the capability to detect very bright EECRs from space.

\section{Acknowledgments}

The work was done with partial financial support from the State Space Corporation ROSCOSMOS, M.V. Lomonosov
Moscow State University through its Prospects for Development program and the Russian Foundation for Basic Research grant No. 16-29-13065 and 15-02-05498/17-a. The Italian group acknowledges financial contribution from the agreement ASI-INAF n.2017-14-H.O and from the Italian Ministry of Foreign Affairs and International Cooperation.

\section{References}

[1] I. Allekotte et al. (Pierre Auger Coll.), Nucl. Instrum. Meth. A 586, 409 (2007).

[2] T. Abu-Zayyad et al. (TA Coll.), Nucl. Instrum. Meth. A 689, 87 (2017).

[3] R. Benson and J. Linsley, International Cosmic Ray Conference, 8, 145 (1981).

[4] R. Streitmatter, Workshop on Observing Giant Cosmic Ray Air Showers From E>10 $20 \mathrm{eV}$ Particles From Space (American Institute of Physics Conference Series, 1998) 95 - 107.

[5] Y. Takahashi, Workshop on Observing Giant Cosmic Ray Air Showers From E>10 $20 \mathrm{eV}$ Particles From Space (American Institute of Physics Conference Series, 1998) 117 - 131.

[6] O. Catalano et al., Nuclear Physics B Proceedings Supplements, 80, 08 (2000).

[7] O. Catalano et al., Astronomy, Cosmology and Fundamental Physics, 427 (2003).

[8] O. Catalano et al., International Cosmic Ray Conference, 2, 1081 (2003).

[9] B. Khrenov, Observing Ultrahigh Energy Cosmic Rays from Space and Earth (American Institute of Physics Conference Series, 2001) 57 - 75.

[10] B. Khrenov et al., Nuclear Physics B Proceedings Supplements, 113/1, 115 (2002).

[11] J.-H. Adams et al. (JEM-EUSO Coll.), Experimental Astronomy, 40, 3 (2015).

[12] G. Abdellaoui et al. (JEM-EUSO Coll.), Astroparticle Physics 10298 (2018).

[13] J.H. Adams et al. (JEM-EUSO Coll.), Experimental Astronomy 40281 (2015).

[14] L. Wiencke and A. Olinto for the JEM-EUSO Coll., PoS(ICRC2017), 1097 (2017).

[15] J.H. Adams et al., ArXiv e-prints [arXiv:1703.04513] (2017).

[16] P. Klimov et al. (Lomonosov-UHECR/TLE Coll.), Space Science Reviews 81 (2017).

[17] F. Capel et al., Advances in Space Research, 10.1016/j.asr.2017.08.030 (2017).

[18] M. Casolino M. et al. (JEM-EUSO Coll.), PoS(ICRC2017) 368 (2017).

[19] A. Olinto et al. (POEMMA Coll.), PoS(ICRC2017) 542 (2017).

[20] B.A. Khrenov et al. (Lomonosov-UHECR/TLE Coll.) J. of Cosmology and Astroparticle Physics 9006 (2017) [arXiv:1704.07704]

[21] P. Klimov et al. (Lomonosov-UHECR/TLE Coll.), Conference Proceedings of $26^{\text {th }}$ ECRS and $35^{\text {th }}$ RCRC, Barnaul \& Belokurikha, Russia (2018). 
$\vartheta=0.00^{\circ} \varphi=0.00^{\circ} \lambda=355.0$ Sim.: 100000 Hit_on_mirror: 77871 (Mir.S $\left[\mathrm{m}^{2}\right]=1.947$ ) Reflected: 56935 ( $73.1 \%$ )

On_PMTs: 50150 Filter_pass: 49318 ( $98.3 \%$

Along $X$ MeantSD: $0.03 \pm 21.02$ Gauss $(X): P e a k \pm \sigma[m m]=:-0.05 \pm 16.53$

Along_Y MeantSD: $0.03 \pm 21.16$ Gauss $(Y): P e a k \pm \sigma[m m]=:-0.01 \pm 15.73$

PRELIMINARY

$1500 \mathrm{~mm}$ : mirror bottom->light guide entrance
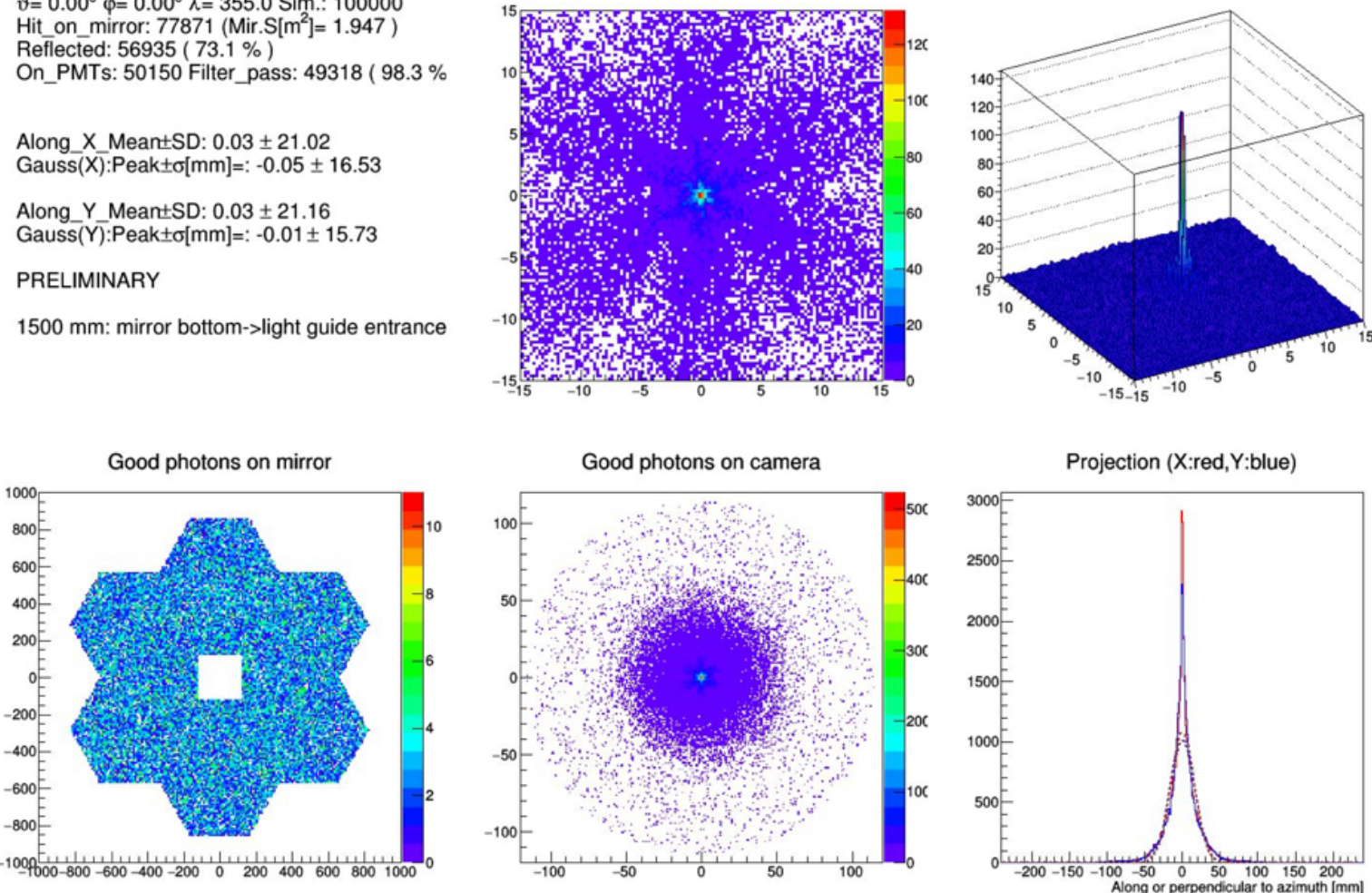

Figure 4. Simulations of the Fresnel mirror response of TUS. The bottom-right plot shows the PSF of the Fresnel optics, which is sharper in the center and broader in the tails compared to a Gaussian shape. Units in the plots are expressed in $\mathrm{mm}$.

[22] P. Klimov et al. (Lomonosov-UHECR/TLE Coll.), These Conference Proceedings (2018).

[23] C. Berat et al., Astrop. Phys. 33/4 221 (2010).

[24] https://gmao.gsfc.nasa.gov/reanalysis/MERRA-2/

[25] https://ngdc.noaa.gov/eog/dmsp.html

[26] M. Zotov for the Lomonosov-UHECR/TLE Coll., poster at the $26^{\text {th }}$ ECRS and $35^{\text {th }}$ RCRC, Barnaul \& Belokurikha, Russia (2018).
[27] P. Klimov et al. (Lomonosov-UHECR/TLE Coll.), PoS(ICRC2017) 1098 (2017).

[28] J.-H. Adams et al. (JEM-EUSO Coll.), Astrop. Phys., 4476 (2013).

[29] M. Bertaina et al. (JEM-EUSO Coll.), Advan. Space Res., 531515 (2014).

[30] N.P. Il'ina et al., Soviet J. Nucl. Phys., 551540 (1992). (2014).

[31] M. Nagano et al., Astrop. Phys., 22235 (2004). 